\title{
Quantum computer inverting time arrow for macroscopic systems
}

\author{
B. Georgeot and D. L. Shepelyansky \\ Laboratoire de Physique Quantique, UMR 5626 du CNRS, Université Paul Sabatier, F-31062 Toulouse Cedex 4, France
}

(May 30, 2001)

A legend tells [1] that once Loschmidt asked Boltzmann on what happens to his statistical theory if one inverts the velocities of all particles, so that, due to the reversibility of Newton's equations, they return from the equilibrium to a nonequilibrium initial state. Boltzmann only replied "then go and invert them". This problem of the relationship between the microscopic and macroscopic descriptions of the physical world and time-reversibility has been hotly debated from the XIXth century up to nowadays [2-9]. At present, no modern computer is able to perform Boltzmann's demand for a macroscopic number of particles. In addition, dynamical chaos 10 13 implies exponential growth of any imprecision in the inversion that leads to practical irreversibility. Here we show that a quantum computer 14 17 composed of a few tens of qubits, and operating even with moderate precision, can perform Boltzmann's demand for a macroscopic number of classical particles. Thus, even in the regime of dynamical chaos, a realistic quantum computer allows to rebuild a specific initial distribution from a macroscopic state given by thermodynamic laws.

To study the relations between microscopic deterministic classical dynamics, macroscopic thermodynamic laws and quantum computation, we choose a simple areapreserving map:

$$
\bar{y}=y+x(\bmod \mathrm{L}), \bar{x}=x+\bar{y}(\bmod 1) .
$$

Here the first equation can be seen as a kick which changes the momentum $y$ of a particle, while the second one corresponds to a free phase rotation in the interval $-0.5 \leq x<0.5$; bars mark the new values of the variables. The map dynamics takes place on a torus of integer length $L$ in the $y$ direction. For $L=1$ this map reduces to the well-known Arnold cat map [10], which describes a fully chaotic dynamics with positive Kolmogorov-Sinai entropy $h \approx 0.96$. As a result, the dynamics is characterized by exponential divergence of nearby trajectories, so that any small error $\epsilon$ (for example round-off error) grows exponentially with time, and reversibility of a trajectory is lost after $t_{E} \approx|\ln \epsilon| / h$ map iterations. For $\epsilon \sim 10^{-8}$ comparable to ordinary precision of the Pentium III, this time scale is rather short $\left(t_{E} \approx 20\right)$. For $L \gg 1$ chaos leads to the diffusive spreading of particles in momentum, which is well described by the Fokker-Planck equation:

$$
\partial w(y, t) / \partial t=D / 2 \quad \partial^{2} w(y, t) / \partial^{2} y,
$$

where the diffusion coefficient $D \approx<x^{2}>=1 / 12$. Thus after a time $t \gg 1 / h$ an initial distribution of particles in (11) evolves towards a Gaussian statistical distribution $w(y, t)=w_{g}(y, t)=\exp \left(-\left(y-y_{0}\right)^{2} /(2 D t)\right) / \sqrt{2 D \pi t}$ with $\left.<y^{2}\right\rangle=D t+y_{0}^{2}$, where $y_{0}=\langle y\rangle$ at $t=0$. On a finite torus this diffusive process relaxes to a homogeneous distribution in $y$ after a time $t_{D} \approx L^{2} / D$.

For the case $L=1$ it was shown that a quantum computer can simulate a discretized version of this map with exponential efficiency [18]. Here we show that for $L \gg 1$ a similar quantum algorithm enables to simulate the evolution of a macroscopic number of classical particles which is governed by the thermodynamic diffusion law. To perform this evolution on a lattice of size $L N^{2}$ (with $N=2^{n_{q}}$ and $L=2^{n_{q^{\prime}}-n_{q}}$ ) this algorithm uses three quantum registers. The first one with $n_{q}$ qubits holds the values of the coordinate $x\left(x_{i}=-0.5+i / N, i=0, \ldots, N-1\right)$, the second one with $n_{q^{\prime}}$ qubits holds the $y$ coordinates $\left(y_{j}=-L / 2+j / N, j=0, \ldots, L N-1\right)$ and the last one with $n_{q^{\prime}}-1$ qubits is used as a workspace. The first two registers describe the discretized classical phase space with $L$ cells and $N^{2}$ points per cell. In this way, the initial positions of $N_{d} \sim N^{2}$ particles can be represented by one quantum state $\sum_{i, j} a_{i j}\left|x_{i}>\right| y_{j}>\mid 0>$, where $a_{i, j}=0$ or $1 / \sqrt{N_{d}}$. The quantum algorithm is based on modular additions performed in a way similar to the one described in 19, through Toffoli and controlled-not gates (CNOT). It requires $10 n_{q}+6 n_{q^{\prime}}-17$ gate operations per map iteration, in contrast to $O\left(2^{2 n_{q}}\right)$ operations for the classical algorithm. The time inversion is also realized by $8 n_{q}+4 n_{q^{\prime}}-13$ gate operations which effectively change $y$ into $-y$ half-way between kicks [18] [20]. In this way, the quantum computer acts in a way similar to Maxwell's demon [21,22] who reverses the velocity of each individual particle.

A perfect quantum computer simulates exactly the map (11), but a realistic physical system always has some imperfections which can destroy time-reversibility. For a classical computer, e. g. Pentium III iterating map (11) round-off errors of amplitude $\epsilon$ destroy the timereversibility of the map dynamics after $t_{E}$ iterations. This fact is illustrated on Fig.1 where it is assumed that the demon inverts the velocities of all trajectories after $t_{r}=35$ iterations with a precision $\epsilon$. After that, the macroscopic distribution starts to return back but after $t_{E} \approx|\ln \epsilon| / h$ iterations the errors become too large and the diffusion process restarts again. In contrast to that, 
on a quantum computer round-off errors can be decreased enormously since the size of the registers grows only linearly with $n_{q}$ and the computation is exponentially efficient. However, a quantum computer has its own natural errors which can be viewed as imprecisions of amplitude $\epsilon$ in the gate operations. The comparison of the two types of errors natural for classical and quantum computers is displayed on Fig.1. It shows that the quantum computation with precision $\epsilon=0.01$ in each gate at each map iteration is able to reverse effectively the diffusion process up to the initial state. That is in striking contrast with the irreversibility of the classical computation with a round-off error of amplitude $\epsilon=10^{-8}$ made only once at $t=t_{r}$ when the demon acts. In this way, the quantum computer succeeds to reverse the thermodynamic diffusive process with enormous number of particles. Indeed, at the moment of inversion $t_{r}$, the distribution of particles is a Gaussian of width $\sigma=\sqrt{2 D t_{r}}$ in agreement with the solution of (2), as is shown in Fig.2.

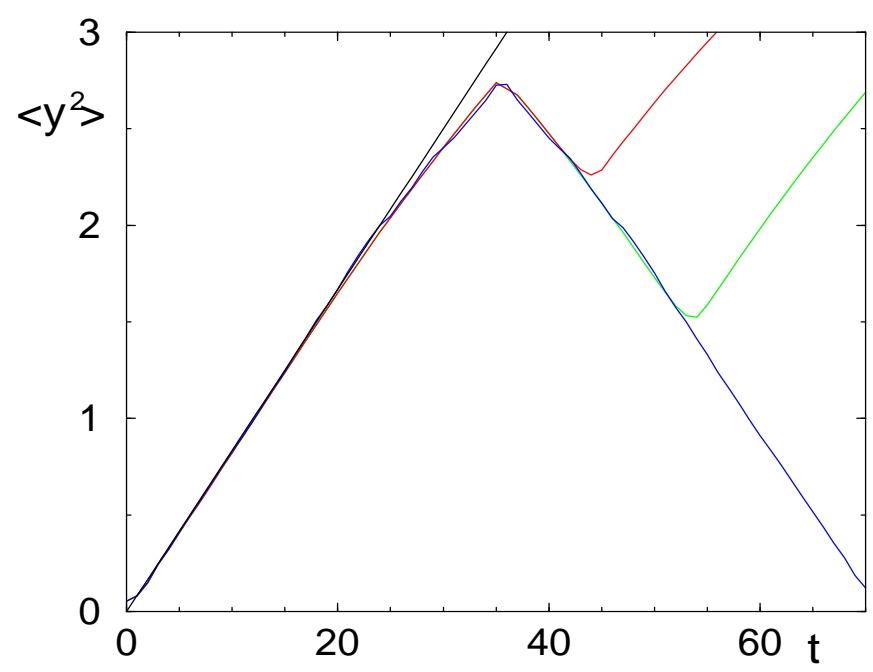

FIG. 1. Diffusive growth of the second moment $\left\langle y^{2}\right\rangle$ of the distribution $w(y, t)$ generated by the map (1) with $L=8$, simulated on a classical (Pentium III) and quantum ("Quantium I") computers. At $t=t_{r}=35$ one inverts all velocities. For Pentium III inversion is done with precision $\epsilon=10^{-4}$ (red line) and $\epsilon=10^{-8}$ (green line); $10^{6}$ orbits are simulated, initially distributed inside the demon image (see Fig. 3). For Quantium I, the computation is done with 26 qubits $\left(n_{q}=7, n_{q^{\prime}}=10\right)$ (blue line); each quantum gate operates with imperfections of amplitude $\epsilon=0.01$ (unitary rotation on a random angle of this amplitude). The black straight line shows the theoretical macroscopic diffusion with $D=1 / 12$.

Fig.3 shows explicitly the distribution in phase space at different moments of time. The initial distribution mimics a demon, which at $t=t_{r}$ is transformed to a statistical homogeneous distribution in the $x$ direction, with a smooth variation in $y$ described by (2). The quantum computer operating with $1 \%$ accuracy is able to recover the initial image with good precision, whereas the classi- cal computer with round-off errors $10^{-8}$ completely fails to reproduce it. The striking difference between the two final distributions at $t=t_{2 r}$ generated by the two computers can be easily detected from a polynomial number of measurements.

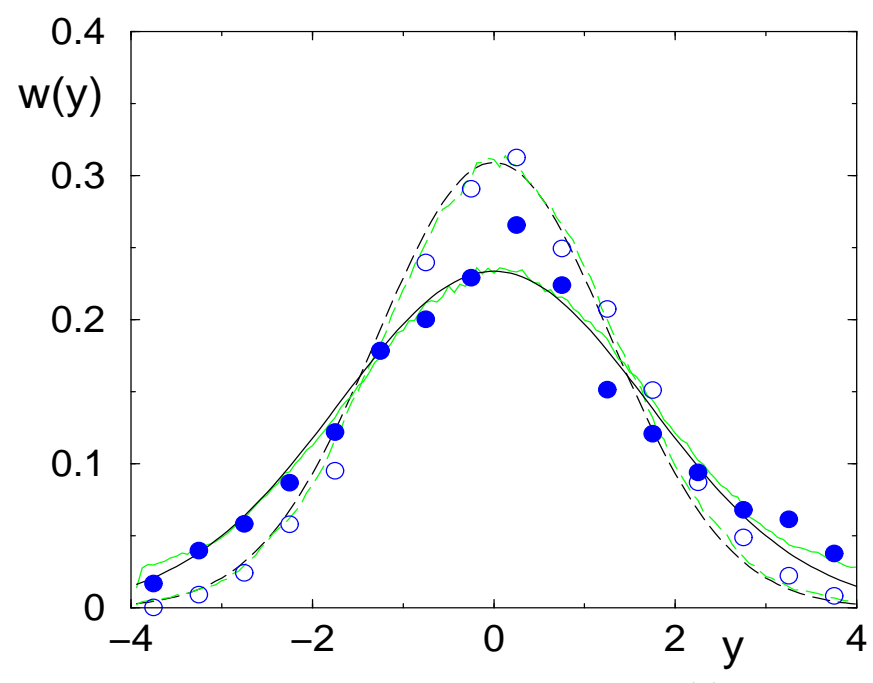

FIG. 2. Distribution of particles in $y$ for map (目) simulated by Pentium III (green lines) and Quantium I (blue symbols) for the case of Fig.1, at $t=20$ (dashed lines and open circles) and $t=t_{r}=35$ (full lines and filled circles). Black lines show the theoretical solution of the Fokker-Planck equation (2).

The previous results are supported by the data for the fidelity $f(t)$ defined as the projection of the quantum state in presence of gate imperfections on the exact state without imperfections. For $f=1$ both states coincide, whereas for $f \ll 1$ both distributions are completely different. The results on Fig.4 show that $f(t)$ smoothly decreases with number of iterations $t$ even if classical dynamics is exponentially unstable. The probability of transition from the exact state to other states induced by imperfections can be estimated as of the order of $\epsilon^{2}$. Hence, since imperfections in each gate are assumed to be uncorrelated, $f(t)$ should drop by $n_{q} \epsilon^{2}$ at each map iteration (for $n_{q} \sim n_{q^{\prime}}$ ). This determines a time scale

$$
t_{f} \approx C /\left(n_{q} \epsilon^{2}\right)
$$

on which the fidelity of quantum computation for the algorithm is reasonable $\left.\left(f\left(t_{f}\right)=0.5\right)\right)$, even in absence of error correction. This scaling is in agreement with the data in Fig.4 (see also [18]) which give the numerical factor $C \approx 0.5$. This is in sharp contrast with classical errors for which computation of trajectories remains correct only up to a time scale $t_{E} \approx|\ln \epsilon| / h$. It is interesting to note that the situation is similar to the time evolution of a physical system in the regime of quantum chaos, which is stable against small quantum errors even though the underlying classical dynamics is chaotic [23. 

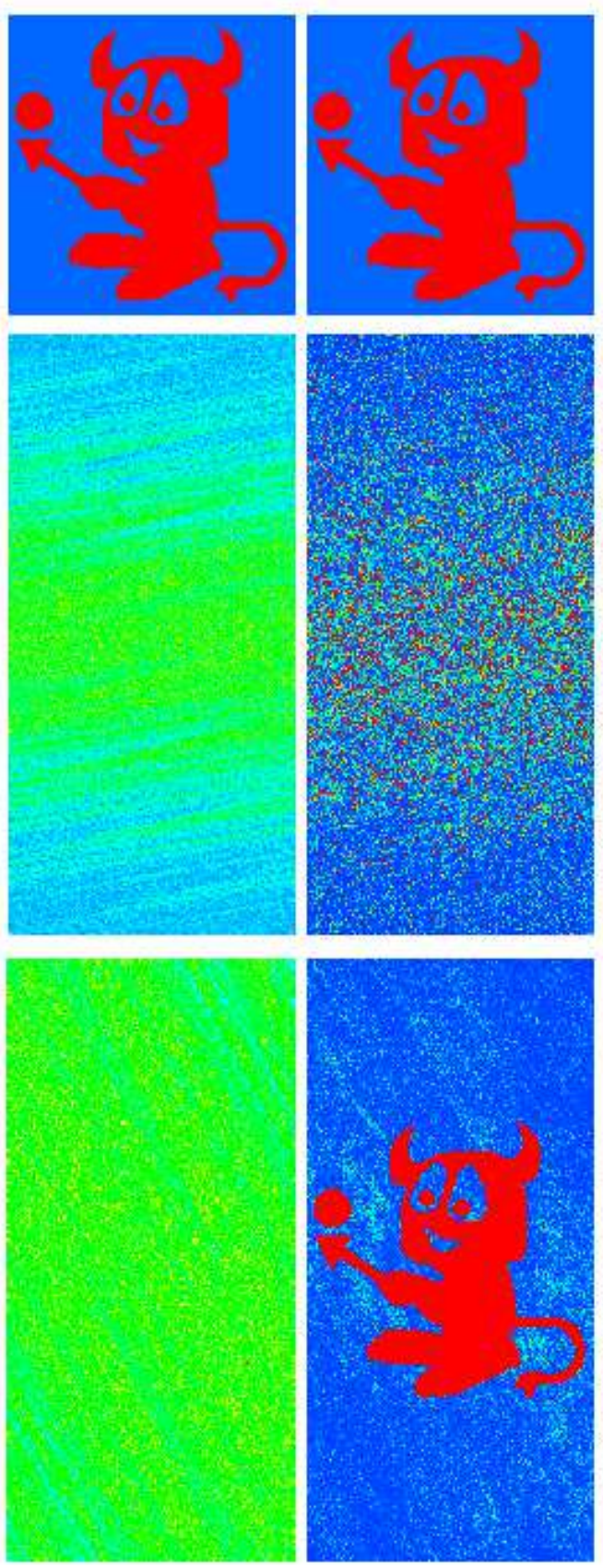

FIG. 3. Evolution of a demon image through map (1). Left column shows the simulation on Pentium III, right column on Quantium I. Top: initial distribution in the central cell $(-0.5 \leq x, y<0.5)$. Middle: distribution at $t=t_{r}=35$ in the whole phase space $(-0.5 \leq x<0.5,-4 \leq y<4)$. Bottom: distribution at $t=2 t_{r}=70$ in the two central cells $(-0.5 \leq x<0.5,-1 \leq y<1)$. The time-inversion is made at $t_{r}=35$, with accuracy $\epsilon=10^{-8}$ for Pentium III (error is done only at $t_{r}$ ), and with accuracy $\epsilon=0.01$ for Quantium I (error is done at each gate operation). Color marks the density of particles/probability, from blue (minimal) to red (maximal value). Here as in Fig.1 $n_{q}=7, n_{q^{\prime}}=10$, with in total 26 qubits used for Quantium I; for Pentium III, $10^{6}$ orbits are simulated.

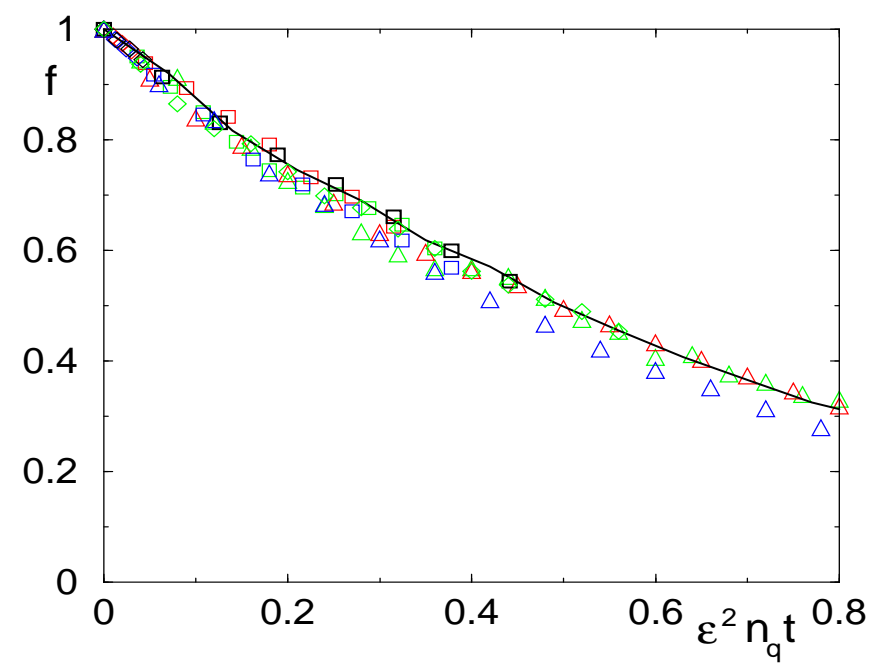

FIG. 4. Fidelity $f$ as a function of $\epsilon^{2} n_{q} t$ for the quantum simulation of the map (11) on Quantium I. Here $L=8$ and $n_{q}=7$, (black line and symbols), $n_{q}=6$ (blue symbols), $n_{q}=5$ (red symbols), $n_{q}=4$ (green symbols)and $\epsilon=10^{-2}$ (diamonds), $\epsilon=3.10^{-2}$ (squares) and $\epsilon=10^{-1}$ (triangles up). Black line shows $n_{q}=7, \epsilon=10^{-1}$.

The relation (3) implies that a quantum computer operating with realistic accuracy can invert velocities of all particles at a given moment of time, so that a specific initial state is reliably reproduced from a statistical distribution described by a diffusive process. Such a simulation for a macroscopic number of particles $N_{d}$ can be performed with few tens of qubits. For example, for $N_{d}=6.022 \times 10^{23}$ (Avogadro's number) the simulation with $L=8$ requires only 125 qubits. Moreover, according to (3), the accurate simulation of such an enormously large number of particles remains reliable $(f(t)>0.5)$ up to a time $t \approx 150$ with a modest gate accuracy $\epsilon=0.01$. Such a computation is far out of reach of any modern supercomputer, and clearly shows the power of quantum computers. It also opens interesting perspectives for cryptography since an initial image can be coded in a thermodynamic distribution with very large entropy and then reliably recovered. Thus quantum computers open new possibilities for the investigation of the relations between microscopic deterministic dynamics and macroscopic thermodynamic laws.

[1] Mayer, J. E. and Goeppert-Mayer, M. Statistical mechanics. (John Wiley \& Sons, N.Y., 1977).

[2] Boltzmann, L. Vorlesungen über Gastheorie, 2 vols. (Barth, Leipzig, 1896, 1898) [English translation Lectures on gas theory (Cambridge University, London, 1964)].

[3] Landauer, R. Irreversibility and heat generation in the computing process. IBM J. Res. Dev. 5, 183 (1961). 
[4] Bennett, C. H. The thermodynamics of computation - a review. Int. J. Theor. Phys. 21, 905 (1982).

[5] Zurek, W. H. Reversibility and stability of information processing systems. Phys. Rev. Lett. 53, 391 (1984).

[6] Lebowitz, J. L. Microscopic origins of irreversible macroscopic behavior. Physica A 263, 516 (1999).

[7] Prigogine, I. Laws of nature, probability and time symmetry breaking. Physica A 263, 528 (1999).

[8] Ruelle, D. Gaps and new ideas in our understanding of nonequilibrium. Physica A 263, 540 (1999).

[9] Hoover, W. G. Time reversibility, computer simulation, and chaos. (World Scientific, Singapore, 1999).

[10] Arnold, V. and Avez, A. Ergodic problems in classical mechanics. (Benjamin, N. Y., 1968).

[11] Kornfeld, I. P., Fomin, S. V. and Sinai, Ya. G. Ergodic theory. (Springer, N. Y., 1982).

[12] Chirikov, B. V. A universal instability of manydimensional oscillator systems. Phys. Rep. 52, 263 (1979).

[13] Lichtenberg, A. and Lieberman, M. Regular and chaotic dynamics. (Springer, N.Y., 1992).

[14] DiVincenzo, D. P. Quantum computation. Science 270, 255 (1995).

[15] Ekert, A. and Josza, R. Quantum computation and Shor's factoring algorithm. Review of Modern Physics 68, 733 (1996).

[16] Steane, A. Quantum computing. Rep. Progr. Phys. 61, 117 (1998).

[17] Kane, B. E. A silicon-based nuclear spin quantum computer. Nature 393, 133 (1998).

[18] Georgeot, B. and Shepelyansky, D. L. Stable quantum computation of unstable classical chaos. Phys. Rev. Lett. 86, 5393 (2001).

[19] Vedral, V., Barenco, A. and Ekert, A. Quantum networks for elementary arithmetic operations. Phys. Rev. A 54, 147 (1996).

[20] The quantum program can be written in the following form for one map iteration, with $\mathrm{C}$ denoting a CNOT gate, $\mathrm{T}$ a Toffoli gate and $\mathrm{R}$ a one-qubit rotation: $\mathrm{T}(\mathrm{TCT})^{n_{q}-1} \mathrm{~T}^{n_{q^{\prime}}-n_{q}-1}(\mathrm{CT})^{n_{q^{\prime}}-n_{q}-1} \mathrm{CTCT}$ $(\mathrm{CCTCT})^{n_{q}-2} \mathrm{CCTCRCC}(\mathrm{TC})^{n_{q^{\prime}}-n_{q}-1}$ $\mathrm{T}^{n_{q^{\prime}}-n_{q}-1} \mathrm{CT}(\mathrm{TCT})^{n_{q}-2}(\mathrm{CCTCT})^{n_{q}-2} \mathrm{CCTC}$ And for the time inversion: $\mathrm{R}^{n_{q^{\prime}}} \mathrm{CT}^{n_{q^{\prime}}-2}(\mathrm{CT})^{n_{q^{\prime}}-2}$ $\mathrm{CCRT}(\mathrm{TCT})^{n_{q}-2}(\mathrm{CCTCT})^{n_{q}-2} \mathrm{CCTC}$

[21] Maxwell, J. C. Theory of heat. (Longmass, Green and Co, London, 1871).

[22] Leff, H. S. and Rex, A.F. Maxwell's demon: entropy, information, computing. (Adam Hilger, Bristol, 1990).

[23] Shepelyansky, D. L. Some statistical properties of simple classically stochastic quantum systems. Physica D 8, 208 (1983).

Acknowledgments: We thank the IDRIS in Orsay and CalMiP in Toulouse for access to their supercomputers, which were used to simulate Quantium I. This work was supported in part by the NSA and ARDA under ARO contract No. DAAD19-01-1-0553 and also by the EC RTN contract HPRN-CT-2000-0156.

Correspondence should be addressed to D.L.Shepelyansky (dima@irsamc.ups-tlse.fr). 